\title{
An automatic wrapped phase gradient-based method for spherical aberration correction in Digital Holographic Microscopy
}

\section{Un método automático basado en el gradiente de la fase envuelta para la corrección de la aberración esférica de fase en microscopía holográfica digital}

\author{
A. C. Monaldi1,2*, G. G. Romero ${ }^{1,2}$, A. V. Blanc ${ }^{2}$, C. M. Cabrera ${ }^{1}$ \\ 1. Universidad Nacional de Salta- Facultad de Ciencias Exactas, Grupo de Óptica Láser \\ 2. INENCO-CONICET \\ ${ }^{(*)}$ E-mail: acmonaldi@gmail.com \\ Received: 01/12/2016 Accepted: 10/07/2018 \\ DOI: 10.7149/OPA.51.3.49032
}

\begin{abstract}
:
Most digital holographic microscopy architectures introduce a microscope objective in the object arm of the micro-interferometer in order to increase lateral resolution but, as a consequence, a spherical phase aberration arises. The phase distortion must be corrected to achieve reliable phase information linked to the microscopic object under study. In this work, we present a fast and simple numerical method for automatically compensate the phase curvature from the wrapped phase gradient of the reconstructed hologram. Since non-unwrapping methods are required, computational cost is significantly reduced. Furthermore, no additional holograms recording is needed and it does not require prior knowledge of the object or the setup. The method is experimentally demonstrated by a phase contrast imaging of a Pediastrum cell.
\end{abstract}

Key words: phase curvature, digital holographic microscopy, Optical phase aberration, wrapped phase

\section{RESUMEN:}

La mayoría de los montajes experimentales utilizados en microscopía holográfica digital introducen un objetivo de microscopio en el brazo objeto del miro-interferómetro con el objetivo de aumentar la resolución lateral; sin embargo, como consecuencia, aparece una aberración de fase esférica que debe corregirse para acceder al retardo de fase que experimenta es espécimen microscópico bajo estudio. En este trabajo se presenta un método rápido y simple para corregir numéricamente la curvatura de fase a partir del gradiente del mapa de fase envuelto del holograma reconstruido. Dado que no se requiere el empleo de métodos de desenvolvimiento de fase, los costos computacionales son notablemente reducidos. Adicionalmente, no se precisa del registro de hologramas adicionales, ni del conocimiento previo del objeto bajo estudio o del arreglo experimental. El método se demuestra experimentalmente a partir del holograma de una microalga del género Pediastrum.

Palabras clave: curvatura de fase, microscopía holográfica digital, aberración óptica de fase, fase envuelta 


\section{REFERENCES AND LINKS / REFERENCIAS Y ENLACES}

[1] C. Mann, L. Yu, C.-M Lo and M. Kim, "High-resolution quantitative phase-contrast microscopy by digital holography”, Opt. Express 13, 8693-8698 (2005).

[2] A. Doblas. D. Hincapie-Zuluaga, G. Saavedra, M. Martínez-Corral and J. García-Sucerquia, "Physical compensation of phase curvature in digital holographic microscopy by use of programmable liquid lens", App. Opt. 54 16, 5229-5233 (2015).

[3] E. Cuche, P. Marquet, and C. Depeursinge, "Simultaneous amplitude-contrast and quantitative phasecontrast microscopy by numerical reconstruction of Fresnel off-axis holograms", Appl. Opt. 38, 6994 (1999).

[4] P. Ferraro, S. De Nicola, A. Finizio, G. Coppola, S. Grilli, C. Magro, and G. Pierattini, "Compensation of the inherent wave front curvature in digital holographic coherent microscopy for quantitative phasecontrast imaging" Appl. Opt. 42, 1938 (2003).

[5] T. Colomb, J. Kühn, F. Charrière, C. Depeursinge, P. Marquet, and N. Aspert, "Total aberrations compensation in digital holographic microscopy with a reference conjugated hologram" Opt. Express 14, 4300 (2006).

[6] T. Colomb, E. Cuche, F. Charrière, J. Kühn, N. Aspert, F. Montfort, P. Marquet, and C. Depeursinge, "Automatic procedure for aberration compensation in digital holographic microscopy and applications to specimen shape compensation", App. Opt. 45 (5), 851-863 (2006).

[7] Tristan Colomb, Frédéric Montfort, Jonas Kühn, Nicolas Aspert, Etienne Cuche, Anca Marian, Florian Charrière, Sébastien Bourquin, Pierre Marquet, and Christian Depeursinge, "Numerical parametric lens for shifting, magnification, and complete aberration compensation in digital holographic microscopy", J Opt Soc Am A 23, 3177-3190 (2006).

[8] J. Di, J. Zhao, W. Sun, H. Jiang, and X. Yan, "Phase aberration compensation of digital holographic microscopy based on least squares surface fitting" Opt. Commun. 282, 3873-3877 (2009).

[9] Y. Wen, W. Qu, C. Cheng, Z. Wang, A. Anand, "Phase errors elimination in compact digital holoscope (CDH) based on a reasonable mathematical model" Pro. of SPIE 9302, 930207 (2015).

[10] L. Miccio, D. Alfieri, S. Grilli, P. Ferraro, A. Finizio, L. De Petrocellis, and S. D. Nicola, "Direct full compensation of the aberrations in quantitative phase microscopy of thin objects by a single digital hologram" Appl. Phys. Lett. 90, 041104 (2007).

[11] C. Zuo, Q. Chen, W. Qu, and A. Asundi, "Phase aberration compensation in digital holographic microscopy based on principal component analysis, Opt. Lett. 38, 1724-1726 (2013).

[12] J. Sun, Q. Chen, Y. Zhang, C. Zuo, “Optimal principal component analysis-based numerical phase aberration compensation method for digital holography” Opt- Lett 41 (6), 1293-1296 (2016).

[13] H. Cui, D. Wang, Y. Wang, J. Zhao and Y. Zhang, "Phase aberration compensation by spectrum centering in digital holographic microscopy", Opt. Commun. 284, 4152-4155 (2011).

[14] J. W. Goodman, Introduction to Fourier Optics, second ed., Mc Graw-Hill Companies, Inc., USA (1996).

[15] A. Monaldi, G. Romero, C. Cabrera, A. Blanc, E. Alanís, "Rolling Shutter Effect Aberration Compensation in Digital Holographic Microscopy”, Opt. Commun 366, 94-98 (2016).

\section{Introduction}

In the last decades, digital holographic microscopy (DHM) has emerged as a powerful tool for studying microscopic samples due to its ability to record and retrieve complete information of the wave transmitted or reflected by a sample from a single hologram. Complex amplitude emerging from the specimen is recorded, via an interferometry setup, by a CCD or CMOS sensor. Then, quantitative phase and amplitude retrieval is performed numerically using scalar diffraction theory foundations. Usually, the light transmitted or reflected by the specimen is collected by a microscope objective (MO) aiming to improve lateral resolution but inducing a phase curvature which needs to be corrected for accurate object phase shift recovery. Several physical and numerical methods have been proposed to compensate this curvature. A simple physical method is achieved by introducing the same curvature in the reference wave using an identical MO at equal distance from the exit of the interferometer [1], but, besides alignment complexity, in off-axis architectures wavefronts perfect matching is not possible, especially when low-power objective lenses are used. Moreover, precise adjustment of the distances to the exit of the interferometer in the measurement process is not easy to achieve in practice. More recently, the use of programmable liquid lens was proposed as a physical alternative to compensate aberration, but prior knowledge of the setup is essential to achieve the correction [2]. Numerical methods accomplish phase compensation during or after 
numerical reconstruction, either in the hologram plane or in the reconstruction plane. Cuche et al [3], for example, used a digital quadratic phase map in the reconstructed plane which needs the manual adjustment of a single parameter that is guessed by trial and error. Every guess involves the reconstruction of the hologram and must be recomputed every time experimental configuration is modified. A double exposure method proposed by Ferraro et al [4] compensates phase curvature completely but requires an additional hologram recording without the samples and a meticulous control of experimental conditions. Similarly, in [5] the correction is done in the hologram plane by using a reference conjugate hologram without the sample or, for setups with reduced aberrations, by the use of the specimen's hologram itself (Self_Reference Conjugated hologram) by low-pass filtering in the frequency domain. However, for this case it is not possible to distinguish between the object and aberration frequency bands. Colomb et al [6,7] proposed to compute phase polynomial masks, called numerical parametric lenses, whose parameters are automatically adjusted by fitting standard polynomials or Zernike polynomials to manual selected 1D profiles or 2D areas extracted from the unwrapped phase where a constant phase is assumed. Others methods correct the curvature automatically by two dimensional fitting of the unwrapped phase signal to a parabolic surface $[8,9]$ or Zernike polynomials [10]. In the Ref. 8, a submatrix is extracted from the unwrapped phase map and then the fitting procedure is performed, but results vary according to the number of sample points, and the criteria to decide a proper value is not automatic. These methods execute least squares fitting without discarding the phase values shifts introduced by the specimen, which generates estimation errors due to the sample itself. More recently, Zuo et al. and Sun et al. proposed a method based on principal component analysis, extracting the aberration term from the first principal component of the phase map, and separating it from the object phase shifts successfully $[11,12]$.

Nevertheless, all mentioned methods exhibit some drawbacks, such as prior knowledge of the setup, manual operation, iterative adjustment, double numerical reconstruction or being computationally expensive and time consuming. Moreover, all of them require unwrapping procedures and, in some cases, phase must be unwrapped at least twice. As a disadvantage, most unwrapping algorithms are very noise sensitive, demand high computational cost or produce errors which are not related with the object or the aberration, and hence they will be propagated to the curvature correction affecting its performance.

In this work, a simple fast and fully automatic method for correcting phase curvature is presented. The compensation is carried out from the wrapped phase gradient map of the reconstructed hologram, which avoids unwrapping procedures and reduces computation time. No prior knowledge of the setup or the object is required.

\section{Principle}

\section{2.a. Experimental setup}

In this study, a transmission DHM system is used, but the principles described are also applicable for reflection mode systems. Details of the experimental setup can be found in Ref. [3]. Briefly, it consists of recording a hologram by means of an interferometric setup, onto a CCD or CMOS sensor, and numerically reconstructing the amplitude and phase of the object wave by means of a computer. In this application, a Mach-Zehnder interferometer is used, whose object arm is fitted with a small microscope built by inserting an $\mathrm{X}-\mathrm{Y}$ microscope stage to locate the sample and a MO which acts as a magnifying lens and forms a real image of the object of interest. The polarized collimated light beam originated at a He-Ne laser of wavelength $\lambda=632.8 \mathrm{~nm}$ is divided by a beam splitter $\mathrm{BS}_{1}$ in order to obtain the object and the reference beams. The plane wave that travels through the object arm, after being reflected by the mirror $M_{1}$, illuminates the sample to be analyzed. The wave diffracted by the specimen, is collected and magnified by an objective lens, $\mathrm{MO}_{1}$. At the same time, the plane wave that travels through the reference arm is reflected by the mirror $\mathrm{M}_{2}$; as mentioned in the previous section, a $\mathrm{MO}_{2}$, with the same characteristics of $\mathrm{MO}_{1}$, can be inserted in the reference arm to partially compensate phase curvature. The object beam and reference beam are recombined in the second beam splitter, $\mathrm{BS}_{2}$, and interfere at the output of the interferometer. A TV camera, with a CMOS Bayer Array 2592 X 1944 pix $^{2}, 1.75 \mu$ m square pixels, 8 bit deep and a frame rate up to $25 \mathrm{~Hz}$ is used to record digital off-axis holograms. A layout of the microscope is depicted in Fig. 1.

If $\mathrm{R}$ is the complex amplitude of the reference beam and $\mathrm{O}$ the complex amplitude of the object beam, then the corresponding intensity distribution at the CMOS sensor can be expressed by the equation:

$$
I_{H}=|R|^{2}+|O|^{2}+R^{*} O+O^{*} R ;
$$


where the symbol * denotes complex conjugate. The first two terms are the zero order, while the third and fourth are the virtual and real images, respectively.

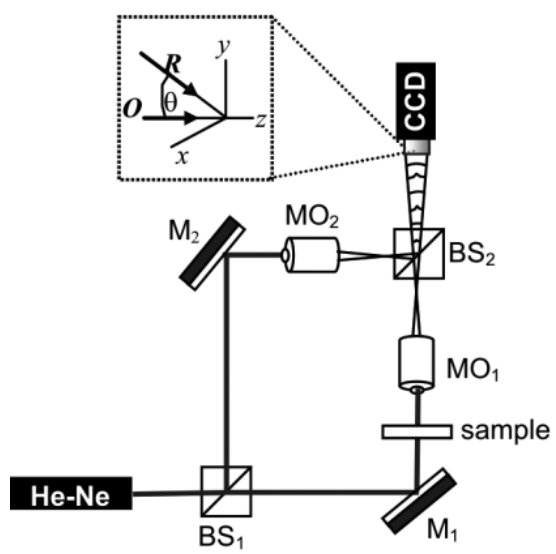

Fig.1. Experimental configuration; BS, beam splitters; M, mirrors; MO, microscope objectives. Inset: R, reference beam; O, object beam.

Off-axis holography is used to allow the separation of the different diffraction orders from a single hologram, but as a consequence, a tilt phase distortion in the reconstructed plane is also introduced. Removing the offaxis tilt can be accomplished by shifting the filtered hologram spectrum $\left(R^{*} O\right)$ to the center of the frequency domain, by locating exactly the position of the carrier frequency of the virtual image [13]. However, the phase curvature is difficult to be completely eliminated, which makes the spectrum centering difficult, mainly due to a fluctuating boundary of the carrier frequency.

\section{2.b. Proposed methodology for Curvature Aberration correction}

If we assume that the MO behaves as a thin lens, delaying the incident wavefront by an amount proportional to the thickness of the lens at each point, then the relationship between the sample (located at the object plane $x_{0} y_{0}$ ), and its magnified image (at the image plane $\xi \eta$ ) is depicted in Fig. 2:

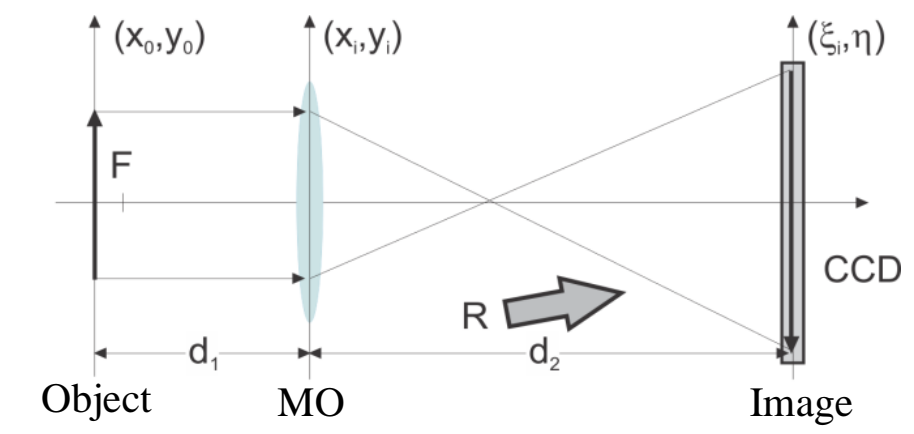

Fig.2. Object and magnified image by an MO in holographic process.

Assuming paraxial approximation, the effects of a thin lens on an incident disturbance is given by the phase transformation [14]:

$$
\exp \left[-\frac{i k}{2 f}\left(x_{i}^{2}+y_{i}^{2}\right)\right]
$$

where $f$ is the focal length and $k=2 \pi / \lambda$ is the wave number, with $\lambda$ the wavelength. This expression may be interpreted as a quadratic approximation to a spherical wave. Thus, a lens composed of spherical surfaces maps an incident plane wave into a spherical wave.

In DHM context, the introduction of a MO in the experimental setup results in an object phase image "mounted" on a parabolic surface which arises from the spherical aberration. In particular, in biological imaging, phase aberration can be significant, but it varies slowly. On the other hand, the phase distribution 
of the specimen is small, but varies fast; thus, it can be regarded as a weak perturbation of the aberration. Hence, the phase of the object wave can be written as:

$$
\Phi(\xi, \eta)=\Phi_{\text {specimen }}(\xi, \eta)+\Phi_{\text {error }}(\xi, \eta) ;
$$

where $\Phi(\xi, \eta)$ is the unwrapped phase retrieved from the holographic reconstruction. It consists of two contributions: $\Phi_{\text {specimen }}(\xi, \eta)$, the ideal phase of the specimen under study, and a weak perturbation of $\Phi_{\text {error }}(\xi, \eta)$, the phase error map encompassing the spherical aberration.

Under this assumption, aberration can be estimated by fitting the reconstructed unwrapped phase to a paraboloid surface, such as:

$$
\Phi_{\text {error }}(\xi, \eta) \sim f(\xi, \eta)=a_{\xi}\left(\xi-b_{\xi}\right)^{2}+a_{\eta}\left(\eta-b_{\eta}\right)^{2}+c ;
$$

where $a_{\xi}, b_{\xi}, a_{\eta}, b_{\eta}$ and $c$ are parameters. Since there are no crossed terms, the coefficients can be identified independently in the $\xi$ and $\eta$ directions; $c$ represents a constant phase over which parabolic surface is mounted. Then, by subtracting the fitted spherical surface from the image wavefront, the corrected image wavefront can be reconstructed including phase information of the specimen under study only.

The main issue of this method is the requirement of performing unwrapping procedures. Besides high computational costs, it is well-known that unwrapped phase may not contain reliable information of the actual phase, and results strongly depend on the algorithm used. Any error in the estimation of the unwrapped phase will be propagated to the fitting algorithm, and thus, to the correction of the aberration.

To overcome this problem, in this work, we propose a method for aberration compensation in which unwrapping procedures are avoided. This is accomplished by accessing to the aberration information from the wrapped phase map itself. From a theoretical point of view, the gradient of the unwrapped phase should be equal to the gradient of the wrapped phase everywhere except in the abrupt discontinuities present in the wrapped phase.

Having into account these ideas, let $\phi_{w}$ represents the wrapped phase map and $\nabla \phi_{w}$ its gradient. If $\Phi_{\text {specimen }}$ is considered as a weak perturbation, gradient of unwrapped phase map $\nabla \Phi$ can be approximated by the gradient of Eq. 4:

$$
\nabla \Phi_{\text {error }} \sim \nabla f=(d f / d \xi, d f / d \eta)=\left(D_{\xi}, D_{\eta}\right)=\left(2 a_{\xi}\left[\xi-b_{\xi}\right], 2 a_{\eta}\left[\eta-b_{\eta}\right]\right)
$$

Accordingly, given the wrapped phase values of $\phi_{w}$, we wish to estimate the aberration $\phi_{\text {error }}$ (Eq. 4) assuming that the gradient of $\phi_{w}$ agrees with the gradient of $\phi_{\text {error }}$ in a least square sense. Therefore, coefficients in Eq. 4 can be obtained without the need of unwrapping the phase.

Therefore, according to Eq. 5, the fitting procedure implies performing a linear regression of $\nabla \phi_{w}$ in the $\xi$ and $\eta$ directions, to the theoretical planes:

$$
A_{\xi} \xi+B_{\xi}=2 a_{\xi}\left[\xi-b_{\xi}\right]
$$

and

$$
A_{\eta} \eta+B_{\eta}=2 a_{\eta}\left[\eta-b_{\eta}\right]
$$

given that $a_{\xi}=A_{\xi} / 2, a_{\eta}=A_{\eta} / 2, b_{\xi}=-B_{\xi} / A_{\xi}$ and $b_{\eta}=-B_{\eta} / A_{\eta}$.

Finally, in order to reduce computational cost even more, instead of a 2D fitting to the planes given by Eq. 6 and 7, a one-dimensional fitting is proposed by extracting two profiles from $\nabla \phi_{w}$ in the $\xi$ and $\eta$ directions. Thus, a 1D fitting is carried out to the straight lines of Eq. 6 and 7. Each profile consists of arranging the median value of each $\xi(\eta)$ coordinate in a 1D array. As an advantage, if the specimen tested spans over an area smaller than the background, it is expected that median values extracted in each direction do not correspond with any value of the object under study. In this case, any phase values shift introduced by the specimen will be discarded, reducing estimation errors due to the sample itself. 


\section{Experimental Results}

For off-axis hologram recording a CMOS sensor Bayer Array 2592x1944 pix ${ }^{2}, 1.75 \mu \mathrm{m}$ square pixels was used. Tilt aberration is corrected by shifting the filtered hologram spectrum to the coordinate origin of the frequency domain. Wavefront reconstruction is carried out by the angular spectrum method [14]. Then, an amplitude and wrapped phase contrast images are obtained. To illustrate the unwanted phase aberration introduced by the MO, in Fig. 3 the reconstructed wrapped phase map obtained from a hologram of a Pediastrum cell immersed in an aqueous medium is shown. As one can observe, the image background is not uniform as it should be according to the homogeneity of the surrounding medium, in this case water. The curvature aberration reveals itself as concentric circles and the density of concentric circles manifests the level of phase aberration. In this particular case, two 20X/0.40 NA objectives were used, one in the object arm and the other in the reference arm of the interferometer. However, as seen in Fig. 3, phase curvature has not been compensated since the distances of the objective lenses from the exit of the interferometer were not equal. In fact, the greater the distance difference from the exit of the interferometer, the greater the phase aberration will be.



Fig.3. Reconstructed wrapped phase of a Pediastrum hologram reveling spherical phase aberration.

It worth noting that some extra aberration appears in Fig. 3; there exists a residual tilt aberration, due to off axis geometry; however, a distortion evidenced as spurious horizontal ripples is also observed. This latter arises from the so called rolling shutter effect and is due to the sequential-readout nature of the CMOS sensor used for recording. A method for correcting this effect was described in Ref. [15].
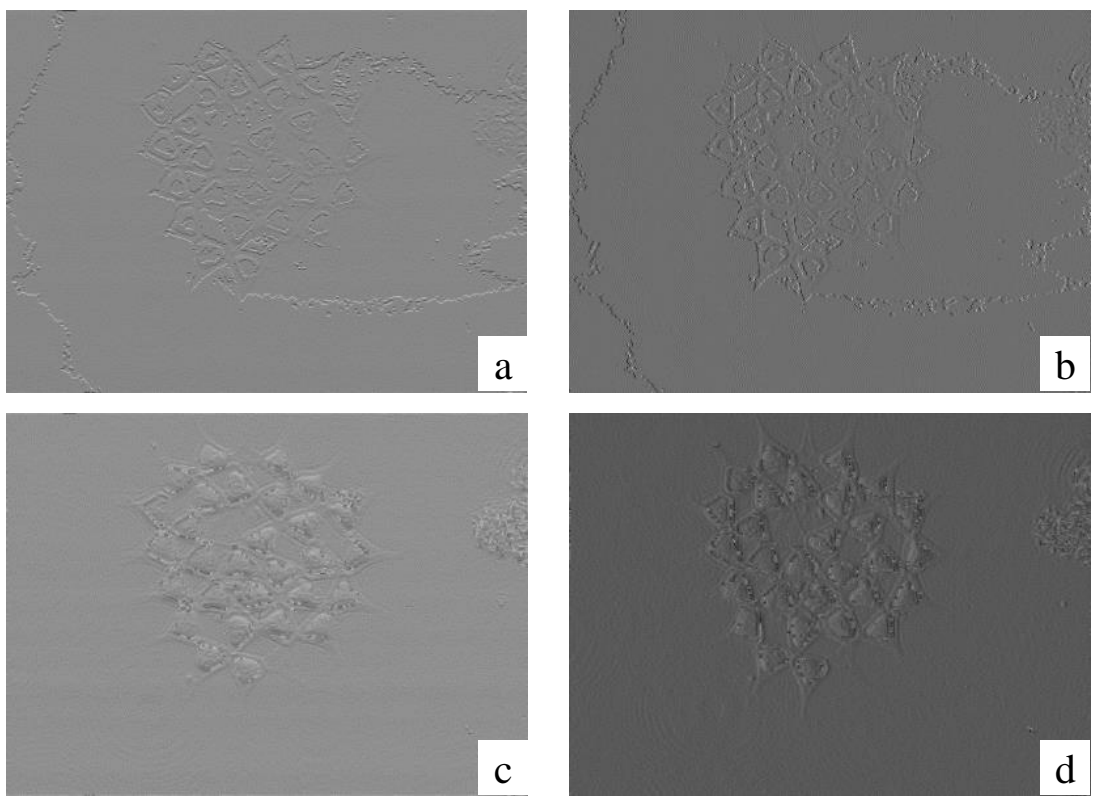

Fig.4. Wrapped phase gradient $\nabla \phi_{w}$ : a) $d \phi_{w} / d \xi$; b) $d \phi_{w} / d \eta$; c) $D_{\xi}^{*}$; d) $D_{\eta}^{*}$.

In order to correct the aberration, the wrapped phase gradient $\nabla \phi_{w}$ is computed in Python by means of NumPy, its fundamental package for scientific computing. Gradient components $d \phi_{w} / d \xi$ and $d \phi_{w} / d \eta$ are shown in Fig. 4 a) and 4 b) respectively. Due to the multiple $2 \pi$ jumps borders of the wrapped phase, the 
gradient map also suffers from large discontinuities in the exact same pixels. In fitting procedure, these discontinuities will act as outliers, and will impact on the fitting performance. To reduce them, each component of $\nabla \phi_{w}$ is wrapped using a wrapping operator $W$ that wraps all values of it argument into the range $(-\pi, \pi)$ by adding or subtracting an integral number of $2 \pi$ radians from its argument, so that $\mathrm{W}\left[\nabla \phi_{w}\right]=$ $\left(D_{\xi}^{*}, D_{\eta}^{*}\right)$. As a result, the two matrixes $D_{\xi}^{*}$ and $D_{\eta}^{*}$ obtained are shown in Fig. $4 \mathrm{c}$ ) and $4 \mathrm{~d}$ ), where it can be seen that the discontinuities due to the spherical aberration have vanished.

As explained in the previous section, these data could be 2D fitted to planes $D_{\xi}$ and $D_{\eta}$ described by Eq. 5, 6 and 7. Instead, the median values of each column of $D_{\xi}^{*}$ and each row of $D_{\eta}^{*}$ are computed and stored in 1D arrays, $\Gamma_{\xi}$ and $\Gamma_{\eta}$ respectively. These arrays incorporate enough information of the aberration and discard any information of the specimen since, in this case, specimen spans over an area smaller than the background. As an example, in Fig. 5 a) the $\Gamma_{\eta}$ profile is depicted, revealing a linear trend corrupted by pronounced oscillations related to the rolling shutter aberration due to the use of the CMOS sensor.

Then, linear regressions of such profiles to the theoretical straight lines given by Eq. 6 and 7 are carried out in order to obtain the adjustment coefficients $a_{\xi}, b_{\xi}, a_{\eta}$ and $b_{\eta}$. To illustrate, the estimated straight line using least squares procedure is also shown in Fig. 5 a). As can be seen, CMOS aberration may strongly affect estimation; therefore, results could be notably improved by correcting this effect [15] or by using a CCD sensor or a CMOS with global shutter as recording devices. The paraboloid of Eq. 4 is obtained straight forward and shown in Fig 5 b).



Fig.5. a) 1D profile $\Gamma_{\eta}$ and linear regression. b) Correction paraboloid.

Finally, the paraboloid is subtracted point by point from the wrapped phase map and wrapped again; the result is illustrated in Fig. 6 b), alongside original wrapped phase (Fig. 6 a)) for comparison purposes. As evidenced, a phase map free of spherical aberration is obtained; compared with Fig. 6 a), it is clear that the phase curvature has been removed from the phase distribution, even with the presence of the unwanted rolling shutter effect.


Fig 6. a) Uncorrected wrapped map. b) Corrected wrapped map.

In order to report a quantitative analysis of the accuracy of the proposed methodology, a hologram without a specimen is recorded. Assuming no air temperature variations, the refractive index is supposed to be uniform over the entire field of view, so the phase signal should be a constant. In Fig. 7 a) and 7 b) the resulting uncorrected and corrected wrapped maps are shown respectively, both corrupted by the rolling shutter effect. Given the horizontal nature of the latter effect, it is not reliable to estimate phase errors by 
computing the standard deviation of a flat area in the phase maps, since they will be affected by this unwanted aberration. In order to compute a measure of the errors induced by the spherical aberration only, phase values of several profiles in the $\mathrm{X}$ direction of the phase image with and without correction are evaluated by a standard deviation computation. As an example, in Fig. 7 c) a horizontal profile and its corrected version are depicted. As expected, when correction is performed, phase is approximately constant. Additionally, when standard deviation values are compared, an average value of 2.13, equivalent to an optical path length $213 \mathrm{~nm}$, is computed for the uncorrected profiles, and an average of 0.34 for the corrected ones. The latter value can be considered as a typical phase error introduced by holographic imaging, which is susceptible to various sources of noise, including quantization noise, shot noise, thermal noise, vibrations and sometimes even speckle.
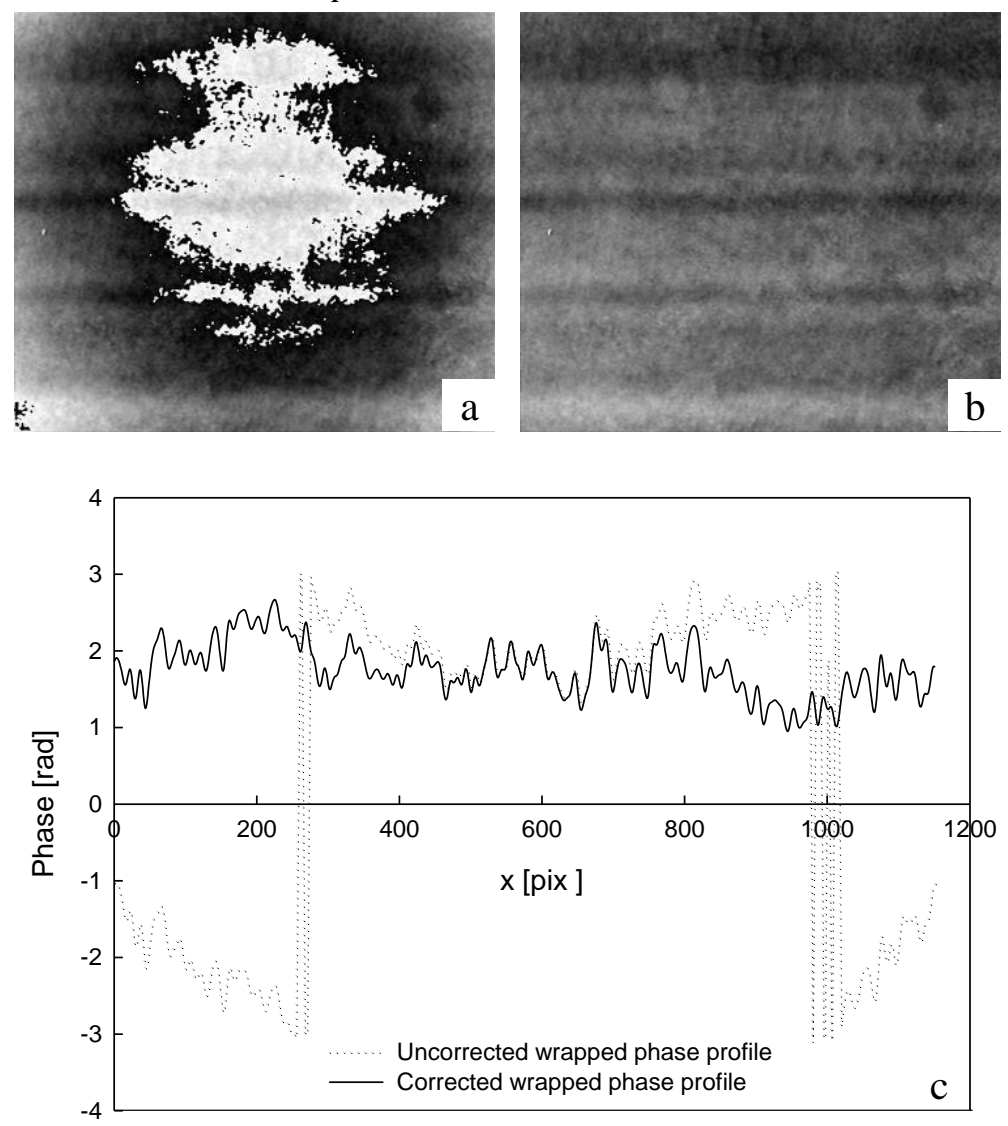

Fig 7. Wrapped signals without specimen a) Uncorrected. b) Corrected. c) An horizontal profile extracted from a) and b)

\section{Conclusions}

Digital holographic microscopy suffers from phase aberrations induced by the imaging system. A simple and fast automatic numerical method for correcting phase curvature introduced by the objective lens has been proposed. The phase aberration is extracted from the wrapped phase gradient. No prior knowledge of the setup was necessary, and no unwrapping algorithms were required. The experiment results show that the proposed method is useful and simple. It is worth emphasizing that the success on the correction was tied to two main assumptions: that specimen phase delays are small compared with the aberration and that spherical aberration can be estimated within the paraxial and thin lens approximation. For high order aberrations (distortion, astigmatism, field curvature, spherical aberration, and so on), the method could be useful inasmuch fitting procedure is carried out with more sophisticated polynomials (such as Zernike polynomials) than the simple one employed in this work.

\section{Acknowledgements}

This work was supported byANPCyT-PME,1392/2 and Research Council of the National University of Salta, Project no. 2161 